\title{
Working Memory Neurons in Pigeons
}

\author{
Bettina Diekamp, Thomas Kalt, and Onur Güntürkün \\ Biopsychologie, Fakultät für Psychologie, Ruhr-Universität Bochum, 44780 Bochum, Germany
}

Working memory, the ability to temporarily store and manipulate currently relevant information, is required for most cognitive faculties. In humans and other mammals, the prefrontal cortex (PFC) provides the underlying neural network for these processes. Within the PFC, working memory neurons display sustained elevated activity while holding active an internal representation of the relevant stimulus during its physical absence or retaining a motor plan for the forthcoming response. Working memory, however, is not a hallmark of higher vertebrates endowed with a neocortex. Birds also master complex cognitive problems invoking working memory, but they lack a laminated neocortex. Behavioral studies in pigeons show that the neostriatum caudolaterale (NCL) plays a central role in executive functions, such as working memory and response control. For neurons in the NCL of pigeons, we show activity changes during the delay of a working memory task, which were similar to those observed in PFC neurons and were related to the successful holding of information in memory and to the subsequent behavior. Thus, although the anatomical and morphological structure of the neuronal substrate in birds is radically different from the mammalian neocortical architecture, the neuronal mechanisms evolved to master equivalent cognitive demands seem to be very similar.

Key words: working memory; birds; forebrain; neostriatum caudolaterale; prefrontal cortex; delayed go-nogo
The prefrontal cortex (PFC) is the essential structure in humans and other mammals for generating working memory (Funahashi and Kubota, 1994; Goldman-Rakic, 1996; Fuster, 1997). Most characteristic of its properties and its role in working memory is the activation of pyramidal cells while stimulus- or task-relevant information is actively maintained over a delay period or while sustaining a motor plan for future actions (Kubota and Niki, 1971; Fuster, 1973; Funahashi et al., 1989; Miller et al., 1996; Rainer et al., 1999; Sawaguchi and Yamane, 1999). Accordingly, prefrontal lesions cause severe deficits in various kinds of working memory tasks (Petrides, 2000; Rowe et al., 2001). Prefrontal functions in cognitive processes also depend on the dopamine system, which exerts modulatory influence onto PFC neurons through dopaminergic innervation from the ventral tegmental area (Arnsten, 1998; Goldman-Rakic, 1999).

Working memory, however, is not a unique ability of higher vertebrates endowed with a neocortex. Birds master numerous tasks invoking working memory (Healy and Krebs, 1992; Mogensen and Divac, 1993; Regolin et al., 1995; Knudsen and Knudsen, 1996). Lesion studies in pigeons have shown that the neostriatum caudolaterale (NCL), a multimodal region in the posterior forebrain, is crucially involved in executive functions, such as working memory and response control (Mogensen and Divac, 1993; Güntürkün, 1997; Hartmann and Güntürkün, 1998; Güntürkün and Durstewitz, 2000). Note that the term "striatum" used in the nomenclature of avian forebrain areas is a misnomer of historical comparative neuroanatomy. The avian neostriatum is not equivalent to the structure with the same name in mammals but is a pallial forebrain component. Functional similarities be-

\footnotetext{
Received Aug. 28, 2001; revised Dec. 4, 2001; accepted Dec. 13, 2001.

This study was supported by the Deutsche Forschungsgemeinschaft. We thank J. Klug, W. Dreckmann, and G. Steinrücke for technical assistance.

Correspondence should be addressed to B. Diekamp, Biopsychologie, Fakultät für Psychologie, GAFO 05/618, Ruhr-Universität Bochum, 44780 Bochum, Germany. E-mail: bettina.diekamp@ruhr-uni-bochum.de.

Copyright (C) 2002 Society for Neuroscience $\quad 0270-6474 / 02 / 220001-05 \$ 15.00 / 0$
}

tween NCL and PFC are evident in the rich dopaminergic innervation from the midbrain (Waldmann and Güntürkün, 1993; Divac et al., 1994; Durstewitz et al., 1999). As in the mammalian PFC, dopamine acting via D1 receptors in the NCL appears to be essential for optimal working memory performance in pigeons (Güntürkün and Durstewitz, 2000; Diekamp et al., 2001). Anatomical studies show correspondence in the pattern of afferent and efferent connections of the NCL and of the mammalian prefrontal system (Kröner and Güntürkün, 1999). Task-related activity of NCL neurons has been described previously in a neurophysiological study, in which pigeons were trained in a go-nogo task (Kalt et al., 1999). However, it is not clear whether the NCL is capable of generating working memory-related activity.

We aimed to investigate the delay activity of single neurons in the NCL of pigeons performing a visual delayed go-nogo task with a well defined working memory component. In this task, the brief presentation of a colored stimulus followed by a short delay instructed the pigeons to execute or withhold beak movements after the delay period. Thus, in this conditional visuomotor task, pigeons were required to either remember the previously presented color during the delay or retain the associated action to manage the task.

This article is published in The Journal of Neuroscience, Rapid Communications Section, which publishes brief, peerreviewed papers online, not in print. Rapid Communications are posted online approximately one month earlier than they would appear if printed. They are listed in the Table of Contents of the next open issue of JNeurosci. Cite this article as: JNeurosci, 2002, 22:RC210 (1-5). The publication date is the date of posting online at www.jneurosci.org.

http://www.jneurosci.org/cgi/content/full/6131 


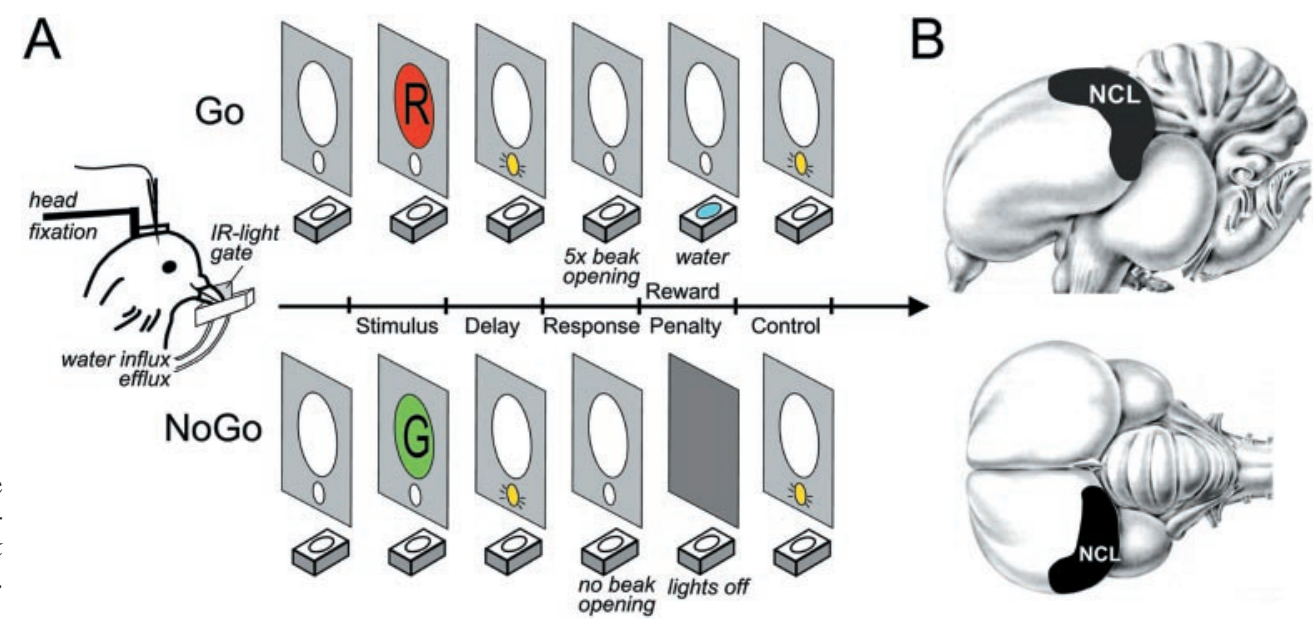

Figure 1. $A$, Sequence of events in the delayed go-nogo task. $B$, Lateral and dorsal view of the pigeons left NCL (black area) from which neurons were recorded. $I R$, Infrared.

\section{MATERIALS AND METHODS}

Subjects. Three adult pigeons (Columba livia) were used in this study. For surgery, they were anesthetized with ketamine $(40 \mathrm{mg} / \mathrm{kg}$, i.m.) and xylazine $(8 \mathrm{mg} / \mathrm{kg}$ i.m. $)$. A recording chamber was positioned and implanted stereotactically over the posterolateral skull overlying the NCL (Karten and Hodos, 1967). In addition, a head-fixation block was implanted medially to the recording chamber. After a few days of recovery, the animals were put on a water control schedule, i.e., animals were deprived of water for 24-36 hr before each training and recording session. Additionally, animals had access to water ad libitum in their home cage for $20 \mathrm{~min}$ after each session. Food was always available ad libitum in their home cage. The procedures were in full compliance with the guidelines of the National Institutes of Health for the care and use of laboratory animals and were approved by a national committee (North Rhine-Westphalia, Germany).

Behavioral task. Pigeons performed a visual delayed go-nogo task (Fig. $1 A$ ). They were restrained by a loose cloth bag and placed on a foam couch in front of a translucent screen. Their head was fixed, and pigeons indicated their response by opening the beak or by suppressing beak openings during the response interval. Movements of the beak were monitored with an infrared light gate. Each trial began with the $500 \mathrm{msec}$ presentation of a colored stimulus (randomly red or green; stimulus size of $35^{\circ}$ of visual angle) on the screen located in front of the pigeon, followed by a delay indicated by a small white light $\left(4^{\circ}\right)$ below the stimulus screen. Both stimuli could be detected by the animal with the eyes placed in lateral viewing position and without shifting gaze. The delay period remained constant in a block of 40-60 trials but varied from 0.8 to $1.6 \mathrm{sec}$ between blocks of trials and sessions, depending on the performance of the individual. At the end of the delay period, the white delay light was switched off, and the pigeon was required to respond within a $2 \mathrm{sec}$ response period. In go trials, the animal had to open and close its beak five times during the response period. Correct responses in go trials ("hit") were rewarded with a small amount of water delivered into a small aluminum container $\left(0.8 \mathrm{~cm}^{3}\right)$ that was placed below their beak. Incorrect go trials ("miss") had no consequences. Beak movements during the response period of nogo trials ("false alarm") led to a mild penalty consisting of a $3 \mathrm{sec}$ time-out with all lights turned off, whereas the suppression of beak movements in nogo trials ("correct rejection") again had no consequences. Thus, to manage the task successfully, pigeons were required to either remember the previously presented color during the delay or form and maintain a motor plan for future actions. To control for the visual stimulation during the delay, the white light was also presented during the intertrial interval, $4.5 \mathrm{sec}$ after the reward or $2.0 \mathrm{sec}$ after the end of the response period. The analysis of behavioral and neural activity in response to the white light during the delay and during this control period was used to assess whether sensory stimulation without working memory load was sufficient to evoke neuronal responses. Trials lasted $\sim 9 \mathrm{sec}$; intertrial intervals were 5-10 sec.

Recording techniques. Extracellular activity was recorded from single cells with glass-insulated platinum-iridium electrodes using standard electrophysiological techniques (Kalt et al., 1999). Neural signals were amplified, filtered, and stored for off-line cluster analysis (DataWave Technologies, Longmont, $\mathrm{CO}$ ) to isolate single units from noise or in a few cases to separate two units recorded from the same electrode. For each neuron, data were sampled over 30-60 go and nogo trials, which were presented in random order.

Data analysis. For each neuron, changes in neuronal activity related to the task were assessed by comparing the spike rates (spikes per second) during specific intervals of the task, using two-tailed $t$ tests for correlated means. Neuronal responses during the delay interval were compared with spontaneous activity (no stimulus, no working memory load) and with spike rates recorded during the control period (identical stimulus, no working memory load). For all neurons, an ANOVA was performed on the average response rates. Different time intervals during each trial (spontaneous activity, stimulus, delay, response, and control interval) and response categories (hit, miss, correct rejection, and false alarm) were used as factors to analyze the effects on the activity of cells. Results were evaluated at $p<0.05$, and, if appropriate, post hoc multiple comparisons (Tukey's test) were applied.

To show the time course of changes in neuronal activity for the different response categories, we calculated normalized average population histograms. Spike counts for each $50 \mathrm{msec}$ bin were normalized by the spontaneous activity of each cell and expressed as a percentage. Separate histograms were calculated for each population of neurons tested with the same delay duration.

To analyze the delay activity in relation to the animal's behavior, we calculated separate Pearson's correlation coefficients between the firing rate of neurons during the delay period and the latency of the fifth mandibulation (leading to the delivery of reward) for each neuron. The correlation coefficients were then subjected to a one-sample Wilcoxon signed rank test to determine whether the mean correlation significantly deviates from zero, indicating a relationship between neuronal delay activity and the response latency of the pigeon.

Histology. On the last day of recordings, electrode tracks were marked with DyeI (Snodderly and Gur, 1995). Animals were deeply anesthetized with Equithesin $(0.31 \mathrm{ml} / 100 \mathrm{gm}$, i.m. $)$ and perfused intracardially. Brains were cut at 40 and $100 \mu \mathrm{m}$, mounted, and stained with cresyl violet and 4',6'-diamidino-2-phenylindole, respectively. Electrode tracks and recording sites were localized in stained sections of the brain by histological verification under fluorescence and light microscopy and by stereotaxic reconstruction. Neuronal data were sampled from locations covering the complete extent of the NCL.

\section{RESULTS}

The pigeons were well trained and reached an average performance of $75 \pm 9 \%$ (mean $\pm \mathrm{SD}$ ) correct trials during recording sessions. Most errors were based on the failure to respond to go trials, i.e., miss trials (20\%), and rarely on false alarms (4\%). While animals were performing the task, extracellular activity of 163 neurons located inside the NCL (Fig. 1B) was recorded. Analysis of neuronal activity revealed that 34 (21\%) of these neurons significantly changed their firing rates during the delay period compared with spontaneous activity ( $t$ tests; all $p<0.05$ ). Neuronal delay activity of these neurons was also significantly different from the activity during the control period. This is proof 


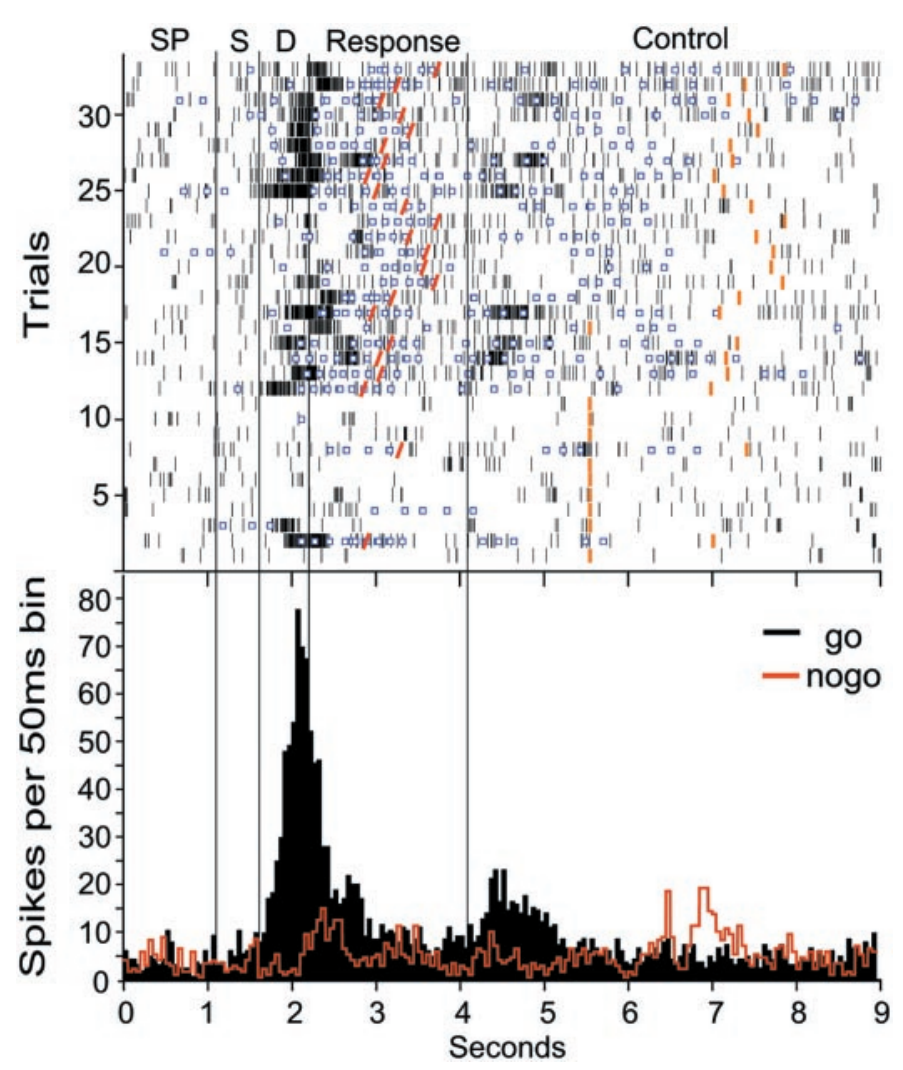

Figure 2. Neuronal activity of a delay neuron. The dot raster (top) is shown only for go trials, and the histogram (bottom) shows the neuronal activity during go (black) and nogo (red) trials. In the raster plot, spikes are indicated by black marks, blue squares indicate beak openings, and red slashes indicate the delivery of water. The vertical lines delineate the spontaneous $(S P)$, stimulus $(S)$, delay $(D)$, and response interval. The beginning of the control interval, which is embedded in the intertrial interval, depends on the animal's behavior and is indicated by orange markers.

that changes in firing frequency were not caused by visual stimulation through the white indicator light during the delay but most likely were related to working memory processes necessary to execute or suppress the behavioral response at the end of the delay period.

The majority of the delay neurons $(n=19)$ expressed enhanced activity during the delay period of go trials, with no changes in firing rates occurring in corresponding nogo trials (Fig. 2). The time course of neuronal activity typically showed a distinct peak in the middle of the delay period or increasing activity toward the end of the delay. The activity of these NCL units was not simply related to the following motor behavior, because the neurons showed no or only minor changes in their discharge rate preceding or during beak movements occurring in the intertrial interval or control period of the task (Fig. 2). Enhanced delay activity in nogo trials was found in two neurons. Thirteen neurons had significantly reduced firing rates during the delay period, six of which were suppressed during the delay of go trials and seven neurons during nogo trials.

An ANOVA performed on all neurons with enhanced delay activity revealed that changes in firing rates were related to the time interval during each trial $\left(F_{(4,72)}=12.5 ; p<0.001\right)$ and response category $\left(F_{(3,54)}=6.4 ; p<0.001\right)$, with a significant interaction between these factors $\left(F_{(12,216)}=7.5 ; p<0.001\right)$ (Fig. $3 A$ ). Neurons showed relatively stable spike rates throughout the entire trial in the case of unsuccessful go trials (miss). The same was true for all nogo trials, in which the animals did (false alarm) or did not (correct rejection) respond. A modulation of activity was only observed in successful go trials (hit), in which spike rates were significantly elevated during the delay interval compared with all other intervals and response categories ( post hoc Tukey's test; $p<0.01$ ). A less pronounced but still significant increase in activity was also evident during the response interval of successful go trials ( $p o s t$ hoc Tukey's test; $p<0.05$ ).

Normalized average histograms of the firing activity of NCL neurons during the task show a clear relationship between the task requirements and the behavior of the animals (Fig. $3 B$ ). Elevated activity during the delay period was observed especially in those trials in which stimulus information or motor actions were successfully retained and in which pigeons were required to activate a motor program. Accordingly, activity patterns during the delay and response period of successful go trials (hit) differed significantly from those of successful nogo trials (correct rejection), when pigeons were not required to activate a motor program and therefore were not necessarily required to memorize the stimulus. Activity changes were less pronounced in miss and false alarm trials. The increase in activity at the beginning of the response period in the case of false alarms might be explained by the activation in preparation of the erroneously performed motor act. The slight enhancement in spike activity during miss trials was probably attributable to the subthreshold activation of the network, which, however, did not suffice to ignite the motor response. Some NCL neurons also showed a minor increase in activity after the response period, which could present an output signal from the NCL.

A relationship between the neuronal activity and the animal's behavior is also evident from the distribution of correlation coefficients between the neuronal firing rate during the delay and the latency of the animal's response (Fig. 3C). The distribution of correlation coefficients significantly differed from zero (onesample Wilcoxon signed rank test; $Z=2.9 ; p<0.004$ ). For the overwhelming majority of neurons, the correlation values were negative, indicating that high neuronal delay activities were followed by a fast behavioral response.

\section{DISCUSSION}

This study, for the first time, shows that the activity of single neurons in birds is modulated during working memory processes. While birds were performing a visual go-nogo task, firing rates of NCL neurons changed during the delay period. This principal pattern of neuronal activation is suitable for carrying information related to the stimulus or to the pending motor response. It is comparable with that recorded from PFC neurons (Kubota and Niki, 1971; Fuster, 1973, Funahashi et al., 1989; Funahashi and Kubota, 1994; Goldman-Rakic, 1996; Miller et al., 1996; Sawaguchi and Yamane, 1999; Rainer et al., 1999) or other associative forebrain areas (Miyashita and Chang, 1988; Quintana and Fuster, 1992) in primates during the delay period of working memory tasks.

In addition, we show that neuronal activity was increased in particular during the delay of successful go trials but not in nogo trials. A relationship between the neuronal discharge during the delay and the behavioral performance of correct versus error trials has also been reported for prefrontal units and the delay task performance in monkeys (Fuster, 1973). In the behavioral paradigm used in the present study, nogo trials may have reduced or no memory demands, because no reward could be earned and 

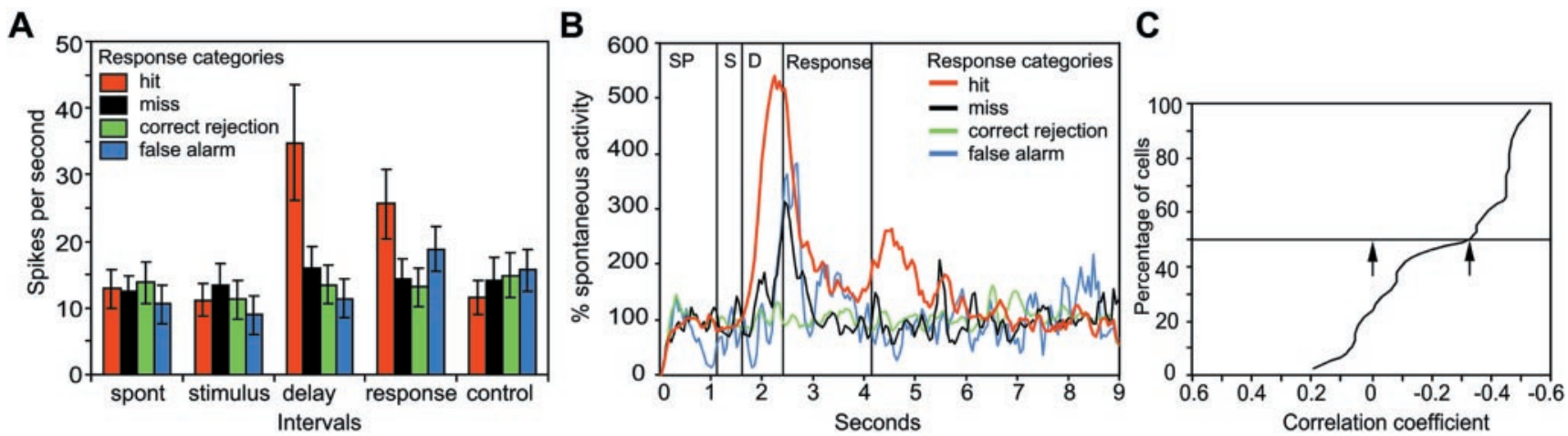

Figure 3. Activity of delay neurons during the go-nogo task. A, Average \pm SEM spike rates across all cells $(n=19)$ with enhanced delay activity for the four response categories and different time intervals during the task in which the average performance was $78 \%$ correct trials. The neural activity of each unit was normalized to spikes per second, taking into account differences in the length of the intervals and the different number of trials that went into each condition. A total of 310 hit, 179 miss, 452 correct rejection, and 34 false alarm trials were analyzed. $B$, Normalized population average histograms for each response category calculated for all neurons $(n=8)$ tested with a $800 \mathrm{msec}$ delay interval show a clear enhancement in activity during the delay of successful go trials. Neural activity is expressed as percentage relative to the spontaneous activity of each cell, which was defined as $100 \%$ and taking into account the different number of trials for each cell in each category. $C$, Cumulative histogram of correlations between neuronal delay activity and latency of the behavioral response leading to reward. Arrows show the median correlation coefficient and the zero value against which the distribution was tested.

no motor response had to be executed after the delay. Thus, the large differences in neural activity observed during the delay of hit trials compared with nogo trials add to the notion that delay activity of NCL neurons indeed represents the neural correlate of working memory. Moreover, it shows that the activity of these neurons during the delay period is not a delayed response to the visual stimulus, because the same colored stimulus elicited disparate responses in hit and miss trials. Neurons within the NCL responding to a visual stimulus while pigeons were performing a similar task had latencies of $<200 \mathrm{msec}$ and responded within the stimulus interval (Kalt et al., 1999).

The smaller changes in neural activity in false alarm trials are most likely related to sensorimotor components of the task and might represent the forthcoming intended movement (Funahashi and Kubota, 1994; Fuster, 1997). Indeed, delay activity of PFC neurons is not only related to the representation of sensory information during working memory but also to the impending motor response (Funahashi and Kubota, 1994; Fuster, 1997; Kim and Shadlen, 1999; Rainer et al., 1999, Constantinidis et al., 2001). However, because motor responses executed by the pigeons in hit trials and false alarm trials were identical, consisting of five beak openings, the much larger observed delay activity in hit trials does not purely reflect the preparation of the motor reaction but, to a large extent, also relates to the sensory representation of the perceived stimulus. Our present data allow no definite answer to which of these representations predominate in the delay activity of NCL neurons. However, in both cases, the discharge bridging the gap between stimulus presentation and motor response is clearly task related and functions in working memory for generating goal-directed behavior. The smaller increase in activity after the response period found in some NCL neurons strongly resembles activity corresponding to output signals recorded from neurons in the mammalian PFC (Funahashi et al., 1989; Funahashi and Kubota, 1994; Fuster, 1997). The relationship between the magnitude of single-unit activity during the delay and the latency of the subsequent response underlines the behavioral relevance of the investigated cellular responses (Rainer et al., 1999). Neuronal activity during the delay period does not merely reflect stimulus attributes but also predicts the reaction time of the operant response.
In summary, the characteristics of neuronal discharge activity in the pigeon NCL during a delay task, as shown in this and a previous study (Kalt et al., 1999), appear to cover all components of working memory, including sensory coding and reward expectancy, mnemonic processes, and motor preparation. Taking into consideration the detrimental effects of NCL lesions on working memory performance (Mogensen and Divac, 1993; Güntürkün, 1997; Hartmann and Güntürkün, 1998) and the neuronal data presented above, the elevated firing patterns observed during the delay period most likely constitute a neuronal correlate of working memory processes in the avian brain.

The discharge properties of NCL neurons might be shaped by the unique properties of the network. Both NCL and PFC are multimodal telencephalic structures defined by a similar set of efferents and afferents, especially from secondary sensory areas and a multisensory thalamic nucleus (Fuster, 1997; Kröner and Güntürkün, 1999). Another distinct feature of this network is its dopaminergic innervation (Waldmann and Güntürkün, 1993; Divac et al., 1994; Durstewitz et al., 1999; Goldman-Rakic, 1999), which modulates neural activity and appears to be crucial for working memory functions and goal-directed behavior (Goldman-Rakic, 1996; Güntürkün and Durstewitz, 2000; Hollerman et al., 2000). Based on these data and lesion studies, showing that the NCL plays a central role in delay and reversal tasks that assess working memory (Mogensen and Divac, 1993; Güntürkün, 1997; Hartmann and Güntürkün, 1998), the NCL has been hypothesized to be functionally similar to the PFC of mammals (Mogensen and Divac, 1993; Divac et al., 1994; Güntürkün, 1997; Hartmann and Güntürkün, 1998; Kröner and Güntürkün, 1999), although NCL and PFC are very likely not homologous structures (Veenman et al., 1997; Kröner and Güntürkün, 1999).

Bearing in mind that the unlayered anatomy of the avian forebrain differs substantially from mammalian neocortex (Durstewitz et al., 1999; Kröner and Güntürkün, 1999) and that genetic expression data during the mammalian neocortical development seem to radically differ from those of the NCL (Puelles et al., 1999), it is tempting to conclude that the observed similarities of neuronal response patterns in NCL and PFC might have evolved independently from each other. As a consequence, equivalent neuronal information processing solutions might have emerged 
when facing related cognitive problems, despite structural differences of the participating neuronal substrates.

\section{REFERENCES}

Arnsten AFT (1998) Catecholamine modulation of prefrontal cortical cognitive function. Trends Cogn Sci 11:436-447.

Constantinidis C, Franowicz MN, Goldman-Rakic PS (2001) The sensory nature of mnemonic representation in the primate prefrontal cortex. Nat Neurosci 4:311-316.

Diekamp B, Kalt T, Ruhm A, Koch M, Güntürkün O (2001) Impairment in a discrimination reversal task after D1-receptor blockade in the pigeon "prefrontal cortex." Behav Neurosci 114:1145-1155.

Divac I, Thibault J, Skageberg G, Palacios JM, Dietl MM (1994) Dopaminergic innervation of the brain in pigeons. The presumed "prefrontal cortex." Acta Neurobiol Exp (Warsz) 54:227-234.

Durstewitz D, Kröner S, Güntürkün O (1999) The dopaminergic innervation of the avian telencephalon. Prog Neurobiol 59:161-195.

Funahashi S, Kubota K (1994) Working memory and prefrontal cortex. Neurosci Res 21:1-11.

Funahashi S, Bruce C, Goldman-Rakic PS (1989) Mnemonic coding of visual space in the monkey's dorsolateral prefrontal cortex. J Neurophysiol 61:331-349.

Fuster JM (1973) Unit activity in prefrontal cortex during delayedresponse performance: neuronal correlates of transient memory. J Neurophysiol 36:61-78.

Fuster JM (1997) The prefrontal cortex: anatomy, physiology, and neurophysiology of the frontal lobe, Ed 3. Philadelphia: Lippincott-Raven.

Goldman-Rakic PS (1996) The prefrontal landscape: implications of functional architecture for understanding human mentation and the central executive. Philos Trans R Soc Lond B Biol Sci 351:1445-1453.

Goldman-Rakic PS (1999) The "psychic" neuron of the cerebral cortex. Ann NY Acad Sci 868:13-26.

Güntürkün O (1997) Cognitive impairments after lesions of the neostriatum caudolaterale and its thalamic afferent in pigeons: functional similarities to the mammalian prefrontal system? J Brain Res 1:133-144.

Güntürkün O, Durstewitz D (2000) Multimodal areas of the avian forebrain-blueprints for cognition? In: Brain evolution and cognition (Roth G, Wullimann M, eds), pp 431-450. Heidelberg: Spektrum Akademischer Verlag.

Hartmann B, Güntürkün O (1998) Selective deficits in reversal learning after neostriatum caudolaterale lesions in pigeons: possible behavioral equivalences to the mammalian prefrontal system. Behav Brain Res 96:125-133.

Healy SD, Krebs JR (1992) Delayed-matching-to-sample by marsh tits and great tits. Q J Exp Psychol [B] 45:33-47.

Hollerman JR, Tremblay L, Schultz W (2000) Involvement of basal ganglia and orbitofrontal cortex in goal-directed behavior. Prog Brain Res 126:193-215.
Kalt T, Diekamp B, Güntürkün O (1999) Single unit activity during a go/nogo task in the "prefrontal cortex" of the pigeon. Brain Res 839:263-278.

Karten HJ, Hodos W (1967) Stereotaxic atlas of the brain of the pigeon (Columba livia). Baltimore: John Hopkins UP.

Kim JN, Shadlen MN (1999) Neural correlates of a decision in the dorsolateral prefrontal cortex of the macaque. Nat Neurosci 2:176-185.

Knudsen EI, Knudsen PF (1996) Disruption of auditory spatial working memory by inactivation of the forebrain archistriatum in barn owls. Nature 383:428-431.

Kröner S, Güntürkün O (1999) Afferent and efferent connections of the caudolateral neostriatum in the pigeon (Columba livia): a retro- and anterograde pathway tracing study. J Comp Neurol 407:228-260.

Kubota K, Niki H (1971) Prefrontal cortical unit activity and delayed alternation performance in monkeys. J Neurophysiol 34:337-347.

Miller EK, Erickson CA, Desimone R (1996) Neural mechanisms of visual working memory in prefrontal cortex of the macaque. J Neurosci 16:5154-5167.

Miyashita Y, Chang HS (1988) Neuronal correlate of pictorial shortterm memory in the primate temporal cortex. Nature 331:68-70.

Mogensen J, Divac I (1993) Behavioural effects of ablation of the pigeon-equivalent of the mammalian prefrontal cortex. Behav Brain Res 55:101-107.

Petrides M (2000) The role of the mid-dorsolateral prefrontal cortex in working memory. Exp Brain Res 133:44-54.

Puelles L, Kuwana E, Puelles E, Rubenstein JL (1999) Comparison of the mammalian and avian telencephalon from the perspective of gene expression data. Eur J Morphol 37:39-50.

Quintana J, Fuster JM (1992) Mnemonic and predictive functions of cortical neurons in a memory task. NeuroReport 3:721-724.

Rainer G, Rao SC, Miller EK (1999) Prospective coding for objects in primate prefrontal cortex. J Neurosci 19:5493-5505.

Regolin L, Vallortigara G, Zanforlin M (1995) Object and spatial representations in detour problems by chicks. Anim Behav 49:195-199.

Rowe AD, Bullock PR, Polkey CE, Morris RG (2001) "Theory of mind" impairments and their relationship to executive functioning following frontal lobe excisions. Brain 124:600-616.

Sawaguchi T, Yamane I (1999) Properties of delay-period neuronal activity in the monkey dorsolateral prefrontal cortex during a spatial delayed matching-to-sample task. J Neurophysiol 82:2070-2080.

Snodderly DM, Gur M (1995) Organization of striate cortex of alert, trained monkeys (Macaca fascicularis): ongoing activity, stimulus selectivity, and widths of receptive field activating regions. J Neurophysiol 74:2100-2125.

Veenman CL, Medina L, Reiner A (1997) Avian homologues of mammalian intralaminar, mediodorsal and midline thalamic nuclei: immunohistochemical and hodological evidence. Brain Behav Evol 49:78-98.

Waldmann C, Güntürkün O (1993) The dopaminergic innervation of the pigeon caudolateral forebrain: immunocytochemical evidence for a "prefrontal cortex" in birds? Brain Res 600:225-234. 